\title{
Fractional flow reserve (FFR)
}

Julian Nam ${ }^{1}$, Andrew Briggs ${ }^{1}$, Jamie Layland ${ }^{2}$, Keith G. Oldroyd ${ }^{3}$, Nick Curzen ${ }^{4}$, Arvind Sood ${ }^{5}$, Kanarath Balachandran ${ }^{6}$, Raj Das ${ }^{7}$, Shahid Junejo ${ }^{8}$, Hany Eteiba ${ }^{3}$, Mark C. Petrie $^{3}$, Mitchell Lindsay ${ }^{3}$, Stuart Watkins ${ }^{3}$, Simon Corbett ${ }^{4}$, Brian O'Rourke ${ }^{5}$, Anna O'Donnell ${ }^{3}$, Andrew Stewart ${ }^{9}$, Andrew Hannah ${ }^{9}$, Alex McConnachie ${ }^{10}$, Robert Henderson ${ }^{11}$ and Colin Berry ${ }^{2,3^{*}}$

\begin{abstract}
Background: In the Fractional flow reserve (FFR) versus angiography in guiding management to optimise outcomes in non-ST elevation myocardial infarction (FAMOUS) clinical trial, FFR was shown to significantly reduce coronary revascularisation, compared to visual interpretation of standard coronary angiography without FFR. We estimated the cost-effectiveness from a UK National Health Service perspective, based on the results of FAMOUS.

Methods: A mixed trial- and model-based approach using decision and statistical modelling was used. Within-trial (1-year) costs and QALYs were assembled at the individual level and then modelled on subsequent management strategy [coronary artery bypass graft (CABG), percutaneous coronary intervention (PCI) or medical therapy (MT)] and major adverse coronary events (death, MI, stroke and revascularisation). One-year resource uses included: material, hospitalisation, medical, health professional service use and events. Utilities were derived from individual EQ5D responses. Unit costs were derived from the literature. Outcomes were extended to a lifetime on the basis of MACE during the 1st year. Costs and QALYS were modelled using generalized linear models whilst MACE was modelled using logistic regression. The analysis adopted a payer perspective. Costs and outcomes were discounted at $3.5 \%$.
\end{abstract}

Results: Costs were related to the subsequent management strategy and MACE whilst QALYs were not. FFR led to a modest cost increase, albeit an imprecise increase, over both the trial [ $£ 112(-£ 129$ to $£ 357)]$ and lifetime horizons [£133 (-£199 to £499)]. FFR led to a small, albeit imprecise, increase in QALYs over both the trial [0.02 ( -0.03 to 0.06)] and lifetime horizons [0.03 (-0.21 to 0.28)]. The mean ICER was $£ 7516 / Q A L Y$ and $£ 4290 / Q A L Y$ over the trial and lifetime horizons, respectively. Decision remained high; FFR had 64 and $59 \%$ probability of cost-effectiveness over trial and lifetime horizons, respectively.

\footnotetext{
*Correspondence: colin.berry@glasgow.ac.uk

${ }^{2}$ Institute of Cardiovascular and Medical Sciences, BHF Glasgow

Cardiovascular Research Centre, University of Glasgow, Glasgow, UK

Full list of author information is available at the end of the article
} 
Conclusions: FFR was cost-effective at the mean, albeit with considerable decision uncertainty. Uncertainty can be reduced with more information on long-term health events.

\section{Background}

Non-ST segment elevation myocardial infarction (NSTEMI) is the most common form of acute coronary syndromes (ACS) [1]. The decision for coronary revascularisation is currently guided by visual interpretation of a coronary angiogram [1-3]. Visual interpretation, however, is subjective, potentially inaccurate and a cause for misdiagnosis and incorrect treatment decisions [4-6].

Myocardial FFR (FFRmyo) is defined as the maximal blood flow to the subtended myocardium in the presence of a stenosis, compared to maximum flow in the absence of a stenosis. An FFR $\leq 0.80$ is correlated with the presence of inducible ischemia whereas an FFR $\geq 0.80$ indicates patients can be managed safely with medical therapy (MT) [7]. Recent studies have demonstrated the value of fractional flow reserve (FFR) in guiding treatment decisions [8-13]. The actual impact of FFR on prospective management strategies has only recently been explored [10]. In addition, it is in patients with unstable coronary artery disease, particularly NSTEMI, who are managed by an invasive treatment strategy where FFR may prove to be clinically most useful.

The Fractional flow reserve versus Angiography in guiding Management to Optimise outcomeS in Non-ST-segment Elevation Myocardial Infarction (FAMOUS-NSTEMI, here referred to as 'FAMOUS') (NCT02073422) was a randomised multicentre pilot trial designed to evaluate impact of FFR vs. coronary angiography without FFR on subsequent management strategy [14] including either percutaneous coronary intervention (PCI), coronary artery bypass graft (CABG) or MT. FAMOUS had a follow-up of 1 year. When FFR results were disclosed in the FFR-guided group, the management strategy changed in $21.6 \%$ of patients and resulted in a higher proportion of management with MT compared to the coronary angiography-guided group.

As FFR is a diagnostic test, the true value of FFR relates to its impact on patient management and outcomes [1517]. This study focuses on the outcomes following patient management both within and beyond the trial time period. The aim of this study was to evaluate the costeffectiveness of FFR compared with standard coronary angiography in patients with NSTEMI.

\section{Methods}

This study expands on the 1-year clinical results of FAMOUS [14] by considering the cost-effectiveness. We used a mixed model- and trial-based approach with decision and statistical modelling. Decision modelling can present patient outcomes as part of the clinical pathway [18, 19]; statistical modelling can identify and reduce heterogeneity [20]. This can be especially important if trial endpoints are then used to estimate final economic endpoints.

The base population was patients with recent NSTEMI (mean 62 years old). The comparators were FFR-guided management and standard angiography-guided management. Economic outcomes were costs and qualityadjusted life-years (QALYs). Outcomes were viewed from a single health payer perspective-namely, the National Health Service (NHS). The analysis was conducted for both the 1-year trial time horizon as well as a lifetime horizon.

\section{Model}

The decision problem is summarised by the following decision analytic model, which was adapted from a previous design [21] (Fig. 1). Health and cost consequences were modelled on the treatment decision (MT, PCI or CABG) because treatment decision was directly informed by FFR or standard angiography. Outcomes were modelled additionally on incident major adverse cardiovascular outcomes (MACE) (death, myocardial infarction, stroke and revascularisation) because MACE served as the intermediate endpoint to model life expectancy beyond the trial time horizon, a common practice in previous models [2, 21]. Following the index year, a common QALY and cost tariff was applied to all years, dependent on the presence of MACE during the index year.

\section{Parameter sources}

The probability of MT, PCI or CABG as well as the probability of MACE following each respectively was derived from the FAMOUS study. The life expectancy beyond 1 year following MACE was derived from a previous model in patients with unstable angina or NSTEMI $[2,21]$.

Trial period resource use was obtained from FAMOUS and unit costs were obtained from the literature. Individual level costs were assembled by multiplying resource use with unit costs. Resource use included: the pressure wire intervention; the treatment strategy (PCI, CABG or medical management); catheterisation laboratory time; Coronary Care Unit (CCU), Intensive Treatment Unit (ITU) and general ward days; other in-hospital procedures 


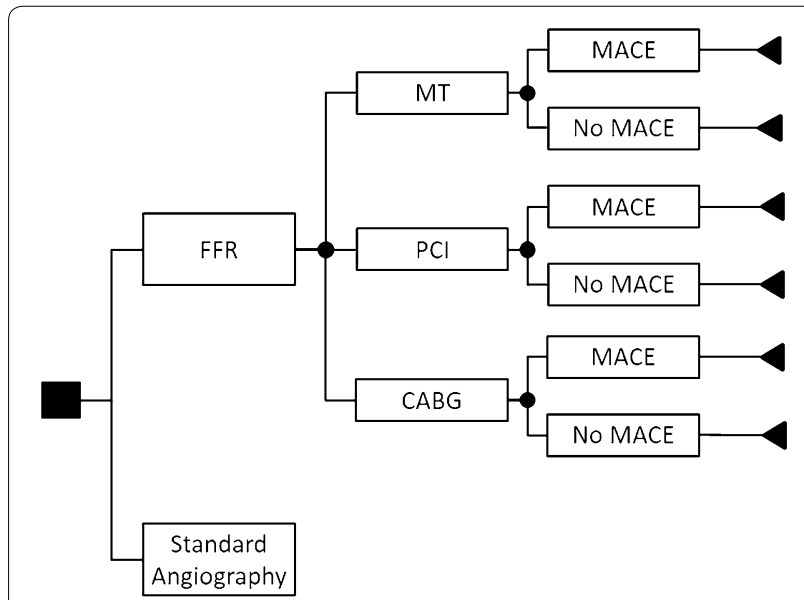

Fig. 1 Model structure showing subsequent management strategy and MACE. Model structure is the same for angiography

(x-rays and echocardiograms) and health events (rehospitalisation, revascularisation, myocardial infarction, and stroke). Regarding the treatment strategy, the analysis included use of PCI materials (catheters, balloons and stents) and drugs (glycoprotein IIa/IIIb inhibitors, bivalirudin, clopidogrel). The use of a pressure wire in patients randomised to coronary angiography alone was removed from the cost estimates as it was protocol driven. Unit cost information was generally derived from national sources, including NHS Reference Costs, the British National Formulary (http://www.bnf.org), Information Services Division Scotland [22] and NICE Clinical Guidelines [2]. Unit cost parameter information is presented in Table 1.

Long-term annual disease costs for those with and without MACE were derived from a previous model of FFR in NSTEMI patients [21].

Trial period QALYs were obtained from FAMOUS by integrating the area under the curve of health utilities. Health utilities at presentation, 6 months and 1 year were estimated with the EuroQol 5D-3L instrument. Responses were converted into utilities with the use of a UK-specific algorithm [23]. For the long-term extension, the utility of those without MACE followed the general population and declined with increasing age in the model. The mean utility change (decrement) of those with MACE was estimated to be $-0.05[21,24]$.

\section{Analysis}

We planned to model one-year trial costs and QALYs on the treatment decision (MT, PCI or CABG) and incident MACE. To estimate the cost parameters, we derived adjusted cost estimates fitting a generalized linear model (GLM) and performing marginal prediction. GLM is the appropriate method when the objective is to obtain efficient estimation of skewed variables [20, 25-27]. Appropriate family and link functions were determined using the Modified Park's and Hosmer and Lemeshow tests, respectively. The regression included a treatment term in addition to other relevant baseline characteristics, selected by a combination of clinical reasoning, independent association with the dependent variable at the $\mathrm{p}<0.25$ level and prevalence $>10 \%$. The final form of the cost model included the following additional covariates: utility at presentation, age, sex, smoking status and history of PCI. The final form of the QALYs model included the following additional covariates: utility at presentation, age, sex and smoking status.

Estimation of MACE following treatment decision (MT, PCI or CABG) was derived in a similar manner. The final form of the model included the following covariates: utility at presentation, age, sex and history of chronic obstructive pulmonary disease. A logistic regression was used to regress MACE on treatment decision, along with baseline characteristics. The ORs for PCI and CABG were then extracted and applied to a baseline MT, which was estimated using marginal prediction. ORs were applied to odds [risk/(1-risk)] and converted to risks (odds/ $(1+$ odds $))$ in the model.

We first tested the significance of the model structure using analysis of variance tests. Partial analysis of variance tests were conducted using deviances from an ANOVA and a $p$ value using the Chi-Squared distribution. If the model structure in Fig. 1 showed no incremental value, raw trial estimates were used.

The developed statistical models were then used to estimate outcomes using marginal prediction. Any observed comparison of two groups, whether randomised or not, is likely to show imbalance in baseline covariates, regardless of statistical significance. Marginal prediction effectively holds all else equal, save for the predictor of interest-treatment.

Missing data was imputed using multiple imputation with chained equations (MICE), where appropriate [28].

We used bootstrapping and probabilistic sensitivity analysis to incorporate sampling uncertainty, parameter uncertainty and model uncertainty. Beta, gamma and lognormal distributions were generally used for utility, cost and relative risk parameters. A dirichlet distribution was used to randomly sample $>2$ rival events, such as the treatment management decision of MT, PCI or CABG. We conducted model selection in Stata 12, statistical modelling in R 3.2 [29] and decision analytic modelling in Microsoft Excel. All costs are presented in 2014 British Pound Sterling.

\section{Research ethics}

The trial was approved by the National Research Ethics Service (reference 11/S0703/6) and complies with the 
Table 1 Data inputs used in the model

\begin{tabular}{|c|c|c|c|c|}
\hline & Mean & SE & Distribution & Source \\
\hline \multicolumn{5}{|l|}{ Treatment decision probabilities } \\
\hline MT, FFR & 0.23 & 0.03 & Dirichlet & FAMOUS \\
\hline $\mathrm{PCl}, \mathrm{FFR}$ & 0.71 & 0.02 & Dirichlet & FAMOUS \\
\hline$C A B G, F F R$ & 0.06 & 0.02 & Dirichlet & FAMOUS \\
\hline MT, standard care & 0.13 & 0.02 & Dirichlet & FAMOUS \\
\hline $\mathrm{PCl}$, standard care & 0.80 & 0.01 & Dirichlet & FAMOUS \\
\hline CABG, standard care & 0.07 & 0.02 & Dirichlet & FAMOUS \\
\hline \multicolumn{5}{|l|}{ Equipment costs $(£)$ : } \\
\hline Guiding catheter & 20 & 0 & - & National procurement \\
\hline Guidewire & 20 & 0 & - & National procurement \\
\hline Pressure wire & 270 & 0 & - & National procurement \\
\hline Adenosine vial & 12 & 0 & - & BNF \\
\hline Balloon catheter & 50 & 0 & - & National procurement \\
\hline Drug eluting stent & 290 & 0 & - & National procurement \\
\hline Bare metal stent & 90 & 0 & - & National procurement \\
\hline Tirofiban (avg/patient) & 242 & 69 & Gamma & BNF; evidence.nhs.uk \\
\hline Bivalirudin (avg/patient) & 625 & 48 & Gamma & BNF; evidence.nhs.uk \\
\hline Clopidogrel (per month) & 2 & & - & BNF; evidence.nhs.uk \\
\hline \multicolumn{5}{|l|}{ Procedure costs $(£)$ : } \\
\hline CABG & 5041 & 313 & Gamma & NHS reference costs \\
\hline Echocardiogram & 128 & 26 & Gamma & Golden Jubilee National Hospital \\
\hline Optical coherence tomography & 1020 & 204 & Gamma & Golden Jubilee National Hospital \\
\hline Intravascular ultrasound & 540 & 108 & Gamma & Golden Jubilee National Hospital \\
\hline Chest x-ray & 18 & 4 & Gamma & Golden Jubilee National Hospital \\
\hline \multicolumn{5}{|l|}{ Hospitalisation costs $(£)$ : } \\
\hline Cath lab time (per hour) & 1681 & 301 & Gamma & ISD Scotland \\
\hline Day in CCU & 1492 & 60 & Gamma & Golden Jubilee National Hospital \\
\hline Day in ITU & 2288 & 458 & Gamma & Golden Jubilee National Hospital \\
\hline Day in general ward & 303 & 17 & Gamma & NHS reference costs \\
\hline \multicolumn{5}{|l|}{ Inhospital event costs $(£)$ : } \\
\hline Severe bleeding & 222 & 3 & Gamma & NHS reference costs \\
\hline Stroke & 2709 & 129 & Gamma & NHS reference costs \\
\hline Ml & 1492 & 76 & Gamma & NHS reference costs \\
\hline \multicolumn{5}{|l|}{ Event costs $(£)$ : } \\
\hline Rehospitalisation & 2261 & 452 & Gamma & NICE CG94 \\
\hline Revascularisation & 2477 & 63 & Gamma & NHS reference costs \\
\hline $\mathrm{Ml}$ & 2261 & 452 & Gamma & NICE CG94 \\
\hline \multicolumn{5}{|l|}{ Disease related costs (index year) (£): } \\
\hline Index year after additional MI & 3228 & 215 & Gamma & NICE CG94 \\
\hline Index year of stroke & 16,926 & 428 & Gamma & NICE CG68 \\
\hline \multicolumn{5}{|l|}{ Additional life-expectancies (years) } \\
\hline MACE & 10.70 & 2.20 & Normal & Kent et al., NICE CG94 \\
\hline No MACE & 5.96 & 1.30 & Normal & Kent et al., NICE CG94 \\
\hline \multicolumn{5}{|l|}{ Other long-term parameters } \\
\hline Annual cost post year $1(£)$ & 423 & 55 & Gamma & Kent et al., NHS reference costs \\
\hline MACE utility change & -0.05 & 0.04 & Beta & Kent et al., Palmer et al. \\
\hline
\end{tabular}


Declaration of Helsinki. The study information sheet that had been approved by the research ethics committee was provided to each participant. Written informed consent was obtained before the diagnostic coronary angiogram and randomization.

\section{Results}

Unadjusted trial outcomes

EQ-5D-3L responses were missing in 17 and $24 \%$ of the trial population at 6- and 12-months, respectively. However, baseline characteristics were balanced across missing and complete groups (Additional file 1); as well, missingness was not statistically associated with intervention (Chi-squared test; $\chi^{2}=0.0234 ; \mathrm{df}=1, \mathrm{p}=0.88$ ). Missing at random was thus a plausible conclusion and multiple imputation was subsequently conducted (Additional file 1).

Following FFR, patients received MT, PCI and CABG with probabilities of 23, 71 and $6 \%$, respectively. Following standard angiography, patients received MT, PCI and CABG with probabilities of 13,80 and $7 \%$, respectively.

Utilities and resource use-unadjusted-for the trial population are tabulated in the Additional file 1. Compared to the standard care group, the utilities in the FFR group were lower at presentation (0.78 vs 0.80$)$ but comparable by 6 -months ( 0.83 vs 0.83$)$ and higher at 12 -months of follow-up ( 0.83 vs. 0.80 ), although none of the differences were significant (Additional file 1).

FFR measurement required an average of 1.03 pressure wires. Lower revascularisation meant lower use of PCI materials such as balloon catheters, drug eluting stents and bare metal stents. Following management with FFR, use of echocardiography was higher whilst OCT and $\mathrm{x}$-rays were no different to standard care. Patients managed with FFR passed similar amounts of time in the catheter laboratory but less ITU time and general ward time. For incident events following treatment, the FFR group had slightly lower rates of rehospitalisation and MI whilst having similar rates of revascularisation, stroke and death compared to standard care; however event rates were extremely low in some and none displayed statistical significance (Additional file 1).

Unadjusted, raw mean costs and QALYs are presented in Table 2. FFR measurement led to increased costs of pressure wire use $(+£ 279)$ and cath lab time $(+£ 57)$ which was offset modestly by savings in PCI $(-£ 92)$, CABG $(-£ 32)$ and medication use $(-£ 17)$. However, the largest cost savings following FFR were in reduced hospital length of stay $(-£ 331)$ and index year events $(-£ 243)$ but they also displayed the greatest uncertainty. Overall, there was a mean cost saving of $£ 349$ and an incremental 0.02 QALYs following FFR during the 1st year.

\section{Statistical models}

Results of the statistical models are provided in the Additional file 1 . Modelling cost on the treatment decision interacted with MACE and displayed statistical significance under a partial analysis of deviance test $(\mathrm{p}<0.001)$; modelling MACE on the treatment decision trended towards statistical significance $(p=0.07)$. For QALYs, however, the addition of treatment decision interacted with MACE did not improve the model $(\mathrm{p}=0.37)$. The model structure in Fig. 1 was thus used for the estimation of cost and MACE whilst it was not imposed for QALYs; instead, unadjusted raw QALYs were used for the trial period.

Table 3 presents the adjusted estimates from the statistical models. As expected, 1-year costs were higher in the presence of MACE vs. no MACE. MT represented the lowest cost, followed by PCI and CABG. Table 3 also presents the extracted odds-ratios of MACE for PCI and

Table 2 Raw unadjusted total and incremental costs and QALYs following standard care and FFR management

\begin{tabular}{|c|c|c|c|}
\hline & $\begin{array}{l}\text { Standard care } \\
\text { Mean }(95 \% \mathrm{Cl})\end{array}$ & $\begin{array}{l}\text { FFR } \\
\text { Mean }(95 \% \mathrm{Cl})\end{array}$ & $\begin{array}{l}\text { Difference } \\
\text { Mean }(95 \% \mathrm{Cl})\end{array}$ \\
\hline \multicolumn{4}{|l|}{ Cost $(£)$} \\
\hline Pressure wire & $0(0-0)$ & $279(273-287)$ & $279(273-287)$ \\
\hline $\mathrm{PCl}$ & $837(750-932)$ & $766(689-844)$ & $-72(-193$ to 45$)$ \\
\hline CABG & $346(174-550)$ & $314(143-487)$ & $-32(-292$ to 226$)$ \\
\hline Medications & $59(48-71)$ & $49(38-60)$ & $-17(-42$ to 7$)$ \\
\hline Cath lab time & 1806 (1672-1949) & $1864(1770-1962)$ & $57(-114$ to 221$)$ \\
\hline Length of stay & 4435 (4043-4847) & $4104(3595-4673)$ & $-331(-1002$ to 356$)$ \\
\hline Other procedures & $125(117-132)$ & $134(127-141)$ & $9(-1$ to 20$)$ \\
\hline Events during index year & $956(613-1372)$ & $713(468-982)$ & -243 ( -736 to 200$)$ \\
\hline Total cost $(£)$ & 8565 (7872-9304) & $8222(7518-8985)$ & $-349(-1367$ to 675$)$ \\
\hline Total QALYs & $0.801(0.765-0.835)$ & $0.82(0.787-0.845)$ & $0.02(-0.029$ to 0.061$)$ \\
\hline
\end{tabular}


Table 3 Statistical model outputs used in the decision analytic model

\begin{tabular}{|c|c|c|}
\hline & Mean & SE \\
\hline \multicolumn{3}{|c|}{ Decision model costs $(£)$} \\
\hline MT, MACE & 9622 & 864 \\
\hline $\mathrm{PCl}, \mathrm{MACE}$ & 14,894 & 589 \\
\hline CABG, MACE & 21,851 & 1984 \\
\hline MT, no MACE & 5819 & 139 \\
\hline $\mathrm{PCl}$, no MACE & 7204 & 72 \\
\hline CABG, no MACE & 17,774 & 482 \\
\hline \multicolumn{3}{|l|}{ MACE parameters } \\
\hline MT (probability) & 0.09 & 0.01 \\
\hline OR for $\mathrm{PCl}$ & 1.01 & $(0.35-2.86)^{\mathrm{a}}$ \\
\hline OR for $C A B G$ & 3.80 & $(0.98-14.62)^{\mathrm{a}}$ \\
\hline
\end{tabular}

a $95 \%$ confidence interval

CABG and the marginal predicted probability of MACE following MT. Compared to MT, PCI did not increase the odds of MACE appreciably, whilst CABG increased the odds over threefold.

\section{Cost-effectiveness}

Mean results of the cost-effectiveness analysis are presented for both the one-year trial and lifetime time horizons (Table 4). FFR led to a mean additional $£ 112$ and 0.01 QALYs over the trial time horizon, compared to standard angiography, whilst over a lifetime it led to an additional $£ 133$ and 0.03 QALYs. The mean ICERs were $£ 7516 /$ QALY and $£ 4290 /$ QALY over the trial and lifetime horizons, respectively.

The cost-effectiveness plane displays the variability in incremental costs and QALYs (Fig. 2). Variability in both incremental costs and QALYs was considerably greater under the lifetime horizon, compared to the trial time horizon. Both time horizons presented consistent results of increased costs against a background of comparable QALYs. This relative comparability in incremental QALYs presents itself more clearly in the cost-effectiveness acceptability curve (CEAC) (Fig. 3). The cost-effectiveness of FFR remained relatively uncertain throughout the willingness-to-pay (WTP) range of $£ 20,000-£ 30,000$ / QALY for both the trial time horizon (64-67\%) and the lifetime horizon ( 59 \%) (Fig. 3).

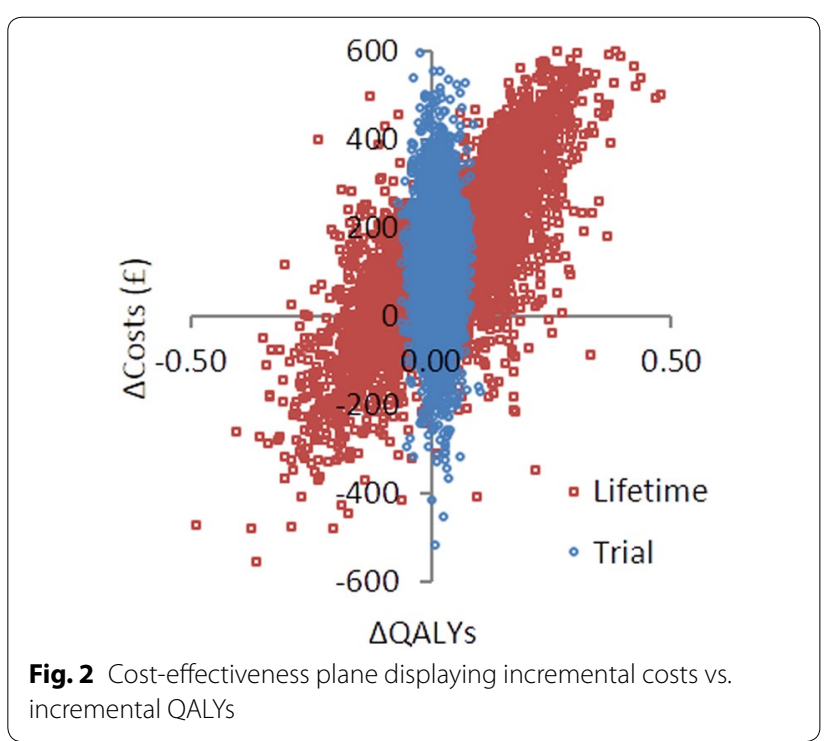

\section{Discussion}

Our analysis presents an early estimate of the combined health and economic impact of FFR-guided management in patients with NSTEMI as measured by FAMOUS. Both the trial and lifetime horizons present consistent results of a modest, relatively precise incremental cost ( $£ 112$ and $£ 133$, respectively) along with modest, albeit imprecise, incremental QALYs (0.01 and 0.03, respectively). FFR displays an acceptable ICER of $£ 4290$ /QALY. However, decision uncertainty remains considerable.

The strength of the present analysis is its use of trial data to reduce heterogeneity in economic outcomes through the use of statistical modelling and marginal prediction. Statistical modelling identified the sources of heterogeneity and distilled the attributable effect of the treatment management (MT, PCI and CABG) along with MACE; one related to the critical decision in the patient management pathway whilst the other related to the critical patient outcomes. Marginal prediction improved estimation of economic outcomes by reducing heterogeneity unrelated to the patient management pathway and critical patient outcomes.

The present results highlight the impact of modelling for within-trial assessments. The impact statistical modelling and marginal prediction on the variability was

Table 4 Results of cost-effectiveness by trial (1-year) and lifetime time horizons. Mean and $95 \% \mathrm{Cl}$ presented

\begin{tabular}{|c|c|c|c|c|c|c|c|}
\hline & \multicolumn{2}{|l|}{ Standard } & \multicolumn{2}{|l|}{ FFR } & \multicolumn{2}{|l|}{ Incremental } & \multirow{2}{*}{$\begin{array}{l}\text { ICER } \\
\text { (£/QALY }\end{array}$} \\
\hline & Costs $(£)$ & QALYs & Costs $(£)$ & QALYs & Costs $(£)$ & QALYs & \\
\hline 71rar & 7574 (6963-8443) & $0.80(0.76-0.84)$ & 7686 (7141-8482) & $0.82(0.79-0.85)$ & $112(-129$ to 357$)$ & $0.01(-0.03$ to 0.06$)$ & 7516 \\
\hline Lifetime & $10,954(9482-12,614)$ & $6.30(5.07-7.23)$ & $11,087(9652-12,699)$ & $6.33(5.09-7.25)$ & 133 (-199 to 499) & $0.03(-0.21$ to 0.28$)$ & 4290 \\
\hline
\end{tabular}




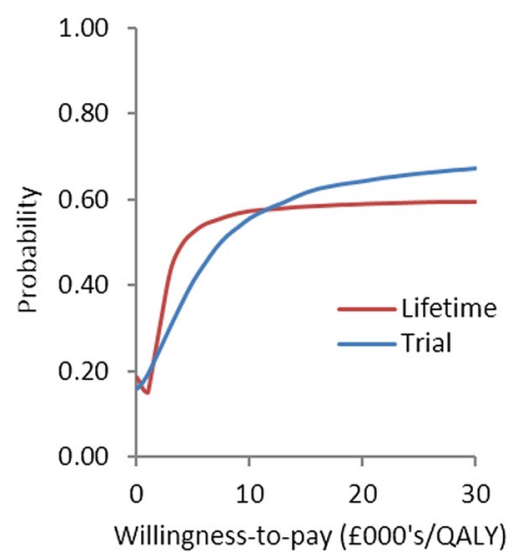

Fig. 3 Cost-effectiveness acceptability curves for the trial and lifetime time horizons

apparent if one compares the variability of trial period costs in the raw vs. modelled results. FFR displayed raw cost-savings during the trial time horizon. Results, however, were very imprecise around a zero difference. By contrast, the decision analysis reduced the $95 \%$ CI nearly six-fold and distilled a more clear direction of the distribution. These adjusted results suggested more modest downstream cost savings that "recouped" part, but not all, of the intervention cost, leading to an overall modest cost increase. As clinical trials becoming an increasingly common source of evidence to assess the value for money of health interventions, analysts should consider such modelling practices.

Downstream cost savings were driven chiefly by absolute, though non-significant, reductions in length of stay and health events such as revascularizations, rehospitalisations, MI and stroke. An improvement of the present analysis would be to model each important outcome separately, rather than as a composite. Composite outcomes make it difficult to attribute the appropriate individual cost and health-related utility tariffs. However, low event counts precluded a more detailed decision model that could characterise health events separately with statistical modelling and marginal prediction.

Inadequate information size was the greatest limitation of the FAMOUS pilot to inform cost-effectiveness. The primary objective of FAMOUS was to gather evidence on the FFR strategy in NSTEMI patients, and although the trial was designed to measure health outcomes at 1 year, it was not powered for these events. The resulting uncertainty was apparent in costs of length of stay and events during the index year, with confidence intervals at least two/threefold greater than other cost categories. Low event counts precluded a more detailed decision model that could characterise downstream MI, rehospitalisation, and other events as functions of the patient management pathway. Low information size also precludes a strong method to extend trial outcomes to a lifetime using the observed data. In order to estimate the wider lifetime impact of FFR, economic modelling would generally extend trial outcomes. This is generally done by extrapolating survival and weighting it with an appropriate quality-of-life utility. However, FAMOUS pilot measured only two time points and observed 8 deaths making any extrapolation uninformative.

The strength of the analysis is also a potential limitation. The decision analysis necessarily applies a structure to the decision problem. We believe the present structure to best represent the patient management pathway and the downstream important patient outcomes. The model structure attributes differences through these pathways. However, it may be the case that differences occur through an alternative pathway. Modelling, however, is unavoidable when extending outcomes beyond the trial horizon. Given the low information size and limited follow-up, the long-term model conditioned life expectancy on MACE. A common utility decrement was applied; however, those with MACE during the index year may return to quality of life similar to those who didn't have MACE, at some point in the future. This would likely increase decision uncertainty by depressing the CEAC further towards $50 \%$. There may be differences in health events between FFR and standard angiography-led management that do not present until well after the index year. At present, there is no indication of this but a larger planned future trial will measure this.

There is only one known study comparing economic outcomes for patients with NSTEMI, by Kent et al. [21]. While their estimates of treatment following FFR were based on data of hypothetical treatment decisions, the present study uses actual decision data from the FAMOUS trial. The hypothetical results underestimated the actual reduction in primary revascularization following FFR (OR 0.88 vs 0.52 , using raw unadjusted trial results). We found comparable incremental QALYs during the index year to the Kent et al. study. We also found FFR led to cost-savings in similar areas, namely index treatment costs and downstream health event costs.

Consistently low mean ICERs over short- and longterm horizons suggest FFR may represent a cost-effective resource allocation over standard angiography. The costeffectiveness of FFR, however, is met with considerable decision uncertainty. Uncertainty can be reduced with more information on long-term health events. Clinical trials are now a common source of evidence to assess the value for money of health interventions. Analysts should consider the benefits of a mixed model- and trial based-approach (or analysis) with decision and statistical modelling. 


\section{Conclusions}

FFR-guided management of NSTEMI may be a costeffective strategy over standard angiography, showing that more targeted invasive management can reduce healthcare resource costs without compromising patient outcomes. However, there still remains considerable decision uncertainty which can be reduced with increased information size and additional long-term evidence on major adverse cardiac events.

\section{Additional file}

Additional file 1. Resource use.

\section{Authors' contributions}

$J N, A B$ and $C B$ designed the economic study. JL, KO, NC, AS, KB, RD, SJ, HE, MCP, ML, SW, SC, BO, AO, AS, AH, AM, RH, and CB designed and conducted FAMOUS. AC conducted the statistical analysis of FAMOUS. JN conducted the economic analysis and wrote the manuscript. $A B$ and $C B$ provided input on the manuscript. All authors read and approved the final manuscript.

\section{Author details}

${ }^{1}$ Health Economics and Health Technology Assessment, University of Glasgow, Glasgow, UK. ${ }^{2}$ Institute of Cardiovascular and Medical Sciences, BHF Glasgow Cardiovascular Research Centre, University of Glasgow, Glasgow, UK. ${ }^{3}$ Department of Cardiology, Golden Jubilee National Hospital, Agamemnon Street, Clydebank G81 4DY, UK. ${ }^{4}$ Department of Cardiology, University Hospital Southampton Foundation Trust, Southampton, UK. ${ }^{5}$ Department of Cardiology, Hairmyres Hospital, East Kilbride, UK. ${ }^{6}$ Department of Cardiology, Royal Blackburn Hospital, Blackburn, UK. ${ }^{7}$ Department of Cardiology, Freeman Hospital, Newcastle, UK. ${ }^{8}$ Department of Cardiology, City Hospitals Sunderland Foundation Trust, Sunderland, UK. ${ }^{9}$ Department of Cardiology, Aberdeen Royal Infirmary, Aberdeen, UK. ${ }^{10}$ Robertson Centre for Biostatistics, University of Glasgow, Glasgow, UK. ${ }^{11}$ Department of Cardiology, Nottingham University Hospitals NHS Trust, Nottingham, UK.

\section{Source of funding}

British Heart Foundation Project Grant PG/11/55/28999; St Jude Medical provided the pressure wires.

\section{Competing interest}

Professor Berry has acted as a consultant for St. Jude Medical based on a contract with his employer, the University of Glasgow. Professor Oldroyd has received consultant and speaker fees from St. Jude Medical and Volcano Corporation which manufacture pressure wires. Professor Curzen has received an unrestricted research grant and fees for lectures and consultancy from St. Jude Medical and an educational grant with Volcano Corporation. None of the other authors have any potential conflict of interest to declare.

Received: 20 July 2015 Accepted: 31 October 2015

Published online: 14 November 2015

\section{References}

1. Hamm CW, Bassand J-P, Agewall S, Bax J, Boersma E, Bueno H, et al. ESC Guidelines for the management of acute coronary syndromes in patients presenting without persistent ST-segment elevation. The task force for the management of acute coronary syndromes (ACS) in patients presenting without persistent ST-segment elevation of the European Society of Cardiology (ESC). Eur Heart J. 2011;32(23):2999-3054.

2. National Institute for Health and Care Excellence. Unstable angina and NSTEMI: the early management of unstable angina and non-ST-segmentelevation myocardial infarction [CG94] [Online]. London: National Institute of Health and Care Excellence; 2010.
3. Wijns W, Kolh P, Danchin N, Di Mario C, Falk V, Folliguet T, et al. Guidelines on myocardial revascularization The task force on myocardial revascularization of the European Society of Cardiology (ESC) and the European Association for Cardio-Thoracic Surgery (EACTS). Eur Heart J. 2010;31(20):2501-55.

4. Botman KJ, Pijls NH, Bech JW, Aarnoudse W, Peels K, van Straten B, et al. Percutaneous coronary intervention or bypass surgery in multivessel disease? A tailored approach based on coronary pressure measurement. Catheter Cardiovasc Interv. 2004;63(2):184-91.

5. Selby JV, Fireman BH, Lundstrom RJ, Swain BE, Truman AF, Wong CC, et al. Variation among hospitals in coronary-angiography practices and outcomes after myocardial infarction in a large health maintenance organization. N Engl J Med. 1996;335(25):1888-96.

6. White CW, Wright CB, Doty DB, Hiratza LF, Eastham CL, Harrison DG, et al. Does visual interpretation of the coronary arteriogram predict the physiologic importance of a coronary stenosis? N Engl J Med. 1984;310(13):819-24.

7. Pijls NH, de Bruyne B, Peels K, van der Voort PH, Bonnier HJ, Bartunek J, et al. Measurement of fractional flow reserve to assess the functional severity of coronary-artery stenoses. N Engl J Med. 1996;334(26):1703-8.

8. Pijls NH, van Schaardenburgh P, Manoharan G, Boersma E, Bech JW, van't Veer $\mathrm{M}$, et al. Percutaneous coronary intervention of functionally nonsignificant stenosis5-year follow-up of the DEFER study. J Am Coll Cardiol. 2007:49(21):2105-11.

9. De Bruyne B, Pijls NH, Bartunek J, Kulecki K, Bech J-W, De Winter H, et al. Fractional flow reserve in patients with prior myocardial infarction. Circulation. 2001;104(2):157-62.

10. Curzen N, Rana O, Golledge P, Zaman A, Oldroyd K, Hanratty C et al. Does routine pressure wire assessment influence management strategy at coronary angiography for diagnosis of chest pain? The RIPCORD Study. Circ Cardiovasc Interv. 2014;7(2):248-55.

11. Tonino PA, De Bruyne B, Pijls NH, Siebert U, Ikeno F, vant Veer M, et al. Fractional flow reserve versus angiography for guiding percutaneous coronary intervention. N Engl J Med. 2009;360(3):213-24.

12. Leesar MA, Abdul-Baki T, Akkus NI, Sharma A, Kannan T, Bolli R. Use of fractional flow reserve versus stress perfusion scintigraphy after unstable angina: effect on duration of hospitalization, cost, procedural characteristics, and clinical outcome. J Am Coll Cardiol. 2003;41(7):1115-21.

13. Sels JWE, Tonino PA, Siebert U, Fearon WF, van't Veer M, de Bruyne B, et al. Fractional flow reserve in unstable angina and non-ST-segment elevation myocardial infarction. experience from the FAME (Fractional flow reserve versus Angiography for Multivessel Evaluation) study. JACC Cardiovasc Interv. 2011;4(11):1183-9.

14. Layland J, Oldroyd KG, Curzen N, Sood A, Balachandran K, Das R et al. Fractional flow reserve vs. angiography in guiding management to optimize outcomes in non-ST-segment elevation myocardial infarction: the British Heart Foundation FAMOUS-NSTEMI randomized trial. Eur Heart J. 2015;36(2):100-11.

15. Bossuyt PM, Irwig L, Craig J, Glasziou P. Comparative accuracy: assessing new tests against existing diagnostic pathways. BMJ. 2006;332(7549):1089-92.

16. di Ruffano LF, Hyde CJ, McCaffery KJ, Bossuyt PM, Deeks JJ. Assessing the value of diagnostic tests: a framework for designing and evaluating trials. Bmj. 2012;344:e686.

17. Schünemann HJ, Oxman AD, Brozek J, Glasziou P, Jaeschke R, Vist GE, et al. Grading quality of evidence and strength of recommendations for diagnostic tests and strategies. BMJ. 2008;336(7653):1106-10.

18. Buxton MJ, Drummond MF, Van Hout BA, Prince RL, Sheldon TA, Szucs T, et al. Modelling in ecomomic evaluation: an unavoidable fact of life. Health Econ. 1997;6(3):217-27.

19. Roberts M, Russell LB, Paltiel AD, Chambers M, McEwan P, Krahn M. Conceptualizing a model a report of the ISPOR-SMDM modeling good research practices task force-2. Med Decis Mak. 2012;32(5):678-89.

20. Glick HA, Doshi JA, Sonnad SS, Polsky D. Economic evaluation in clinical trials. Oxford: Oxford University Press; 2014.

21. Kent S, Briggs A, Eckermann S, Berry C. Are value of information methods ready for prime time? An application to alternative treatment strategies for NSTEMI patients. Int J Technol Assess Health Care. 2013;29(04):435-42.

22. Information Services Division Scotland. Theatre-direct cost per hour, by specialty [R142X] [Online]. Scotland: ISD Scotland; 2012. 
23. Dolan P. Modeling valuations for EuroQol health states. Med Care. 1997;35(11):1095-108.

24. Palmer S, Sculpher M, Philips Z, Robinson M, Ginnelly L, Bakhai A, et al. Management of non-ST-elevation acute coronary syndromes: how costeffective are glycoprotein IIb/llla antagonists in the UK National Health Service? Int J Cardiol. 2005;100(2):229-40

25. Manning WG, Mullahy J. Estimating log models: to transform or not to transform? J Health Econ. 2001;20(4):461-94.
26. Manning WG, Basu A, Mullahy J. Generalized modeling approaches to risk adjustment of skewed outcomes data. J Health Econ. 2005;24(3):465-88

27. Barber J, Thompson S. Multiple regression of cost data: use of generalised linear models. J Health Serv Res Policy. 2004;9(4):197-204.

28. Van Buuren S, Groothuis-Oudshoorn K. MICE: Multivariate imputation by chained equations in R. J Stat Softw. 2011;45(3):1-67.

29. R Core Team. R: a language and environment for statistical computing. Vienna: R Foundation for Statistical Computing; 2015.

\section{Submit your next manuscript to BioMed Central} and take full advantage of:

- Convenient online submission

- Thorough peer review

- No space constraints or color figure charges

- Immediate publication on acceptance

- Inclusion in PubMed, CAS, Scopus and Google Scholar

- Research which is freely available for redistribution

Submit your manuscript at

www.biomedcentral.com/submit

Biomed Central 